\title{
Simulation on adjacent line mutual interference of high-speed railway track circuit
}

\author{
Qihui Xiong, Shiwu Yang*, and Chang Liu \\ ${ }^{1}$ School of Electronic and Information Engineering, Beijing Jiaotong University, No.3, Shangyuan \\ Village, Haidian District, 100044 Beijing, China
}

\begin{abstract}
As the density of the high-speed railway network continues to increase, the problem of electromagnetic interference on adjacent lines has become increasingly prominent. This paper focuses on the electromagnetic interference of adjacent lines caused by rail and line in the signal transmission process of the high-speed rail track circuit. Firstly, complete the establishment of the four-terminal network model of the ZPW-2000A track circuit system and the cab signal entry current crosstalk model, calculation of interference voltage under different parallel length of signal frequency. Then the interference factors and coupling mechanism of adjacent lines are analysed to realize calculation of interference amount. Finally, according to the sensitivity index of the cab signal, the maximum parallel length of adjacent sections is given respect, and the interference protection suggestions of adjacent lines are put forward. The research work of this paper provides a theoretical basis for suppressing the interference of adjacent lines and guarantees the safe and efficient operation of high-speed trains.
\end{abstract}

Keywords: High-speed railway, ZPW-2000A track circuit, Adjacent line interference, Rail mutual inductance, Inductive coupling.

\section{Introduction}

The track circuit is an important device for detecting train occupancy, rail integrity, and train-ground information transmission in the railway signal system. Its reliability is directly related to the safety and efficiency of trains. In the high-speed and heavy-haul electrified railway, the track circuit is affected by the surrounding strong electromagnetic interference sources (such as the traction power supply system) [1]. Due to the increase in the density of the road network, the interference between adjacent lines in the railway site shows the characteristics of irregular interference waveforms, uncertain sources of interference, and difficulty incomplete elimination, which is easy to cause missing or upgrading cab signal code [2]. Therefore, it is significance to establish the simulation model of adjacent line interference of track circuit to realize the protection of electromagnetic interference of railway signal system to ensure the safe and efficient operation of trains.

\footnotetext{
*Corresponding author: ysw@bjtu.edu.cn
} 
As a traditional and inherent problem of railway signals, adjacent line interference has always attracted the attention of domestic and foreign researchers. Lucca[3] et al. studied the influence of inductive interference caused by the electrified double track on the track circuit, and proposed an algorithm for sensitivity analysis of the electromagnetic interference level generated; E. M. Tarasov[4] et al. proposed a method to determine the parameter matrix coefficients of railway multiple lines and analyzed the adjacent line interference between asymmetrical track circuits; Manakov $\mathrm{AD}[5]$ et al. proposed a method to determine the interference current of the double-track circuit; Cuiqin Zhao[6] et al. established a multi-line parallel ballasted track coupling interference equivalent circuit model; Yuequan Li[7] et al. studied the coupling interference at the transverse connecting line at the entrance of electrified railway; Jialiang Liu[8] and others realized the numerical calculation of interference current in the adjacent segment. Most of the above-mentioned research is based on general-speed railways, while high-speed railways have different configurations. And in the face of more stringent safety requirements and a more complex electromagnetic environment, the mechanism of adjacent line interference is still worthy of further study.

\section{Overview of adjacent track circuit interference factors}

The track circuit of this line can receive not only the signal of this line but also the track circuit signal of adjacent lines. When analyzing the interference problem of adjacent lines, the track circuit of this line is called the interfered loop, and the track circuit of adjacent lines is the interfering loop.

China's high-speed railway mainly uses ZPW-2000 series track circuits, with a carrier frequency ranging from 1700 to $2600 \mathrm{~Hz}$. Because the signal current flowing through the track circuit is a high carrier frequency, and the inductive characteristics of the rail, the varying signal current in the interfering loop forms an interference signal in the interfered loop through mutual inductance between rails, which is called mutual inductance coupling. The existence of capacitance between parallel lines makes the energy of other lines couple to interfered loop, which is called capacitive coupling. The current in the rail will leak to another rail through the track bed, forming many parallel leakage channels between the interfering loop and the interfered loop, so that the signal current of the interfering loop is conducted to the interfered loop via the ballast leakage resistance, which is called Ballast resistance leakage conduction. Under actual conditions on-site, due to factors such as humidity, rainfall, snowfall, etc., the resistance of the ballast is reduced, and the leakage of the ballast bed is more serious, which not only causes the red band phenomenon in this track section but also makes the adjacent line interference problem more serious. In the shunt state, the shunt current is relatively large, and the problem of adjacent line interference is particularly complicated.

To sum up, mutual inductance coupling, capacitance coupling, ballast resistance leakage conduction and other electromagnetic interference work together, resulting in adjacent line interference problem. When the interference is serious, it may cause distortion of cab signal receiving current waveform, affecting the normal operation of the track circuit. 


\section{Modelling and analysis of adjacent line interference in co- directional non-insulated track circuit}

\subsection{Modelling and verification of ZPW-2000A track circuit}

The track circuit can be regarded as an electrical circuit with uniformly distributed parameters. Generally, the uniform transmission line theory is used to establish a four-port network model for analysis and calculation. This paper has completed the modelling and simulation of ZPW-2000A type non-insulated track circuit, and considers the mutual crosstalk system model of adjacent jointless track circuits in the same direction. And analyze the influence of electromagnetic interference caused by mutual inductance coupling, capacitive coupling, and track bed leakage conduction on cab signals.

According to the structural characteristics of the ZPW-2000A track circuit, a hierarchical four-port network model of component level, module level, and system level is established as shown in Figure 1. In shunt state, because the transient component of the signal attenuates quickly when the train slides on the track circuit, it is only necessary to consider the cascaded shunt resistance $R_{f}$ (taking $0.15 \Omega$ ) at different positions.

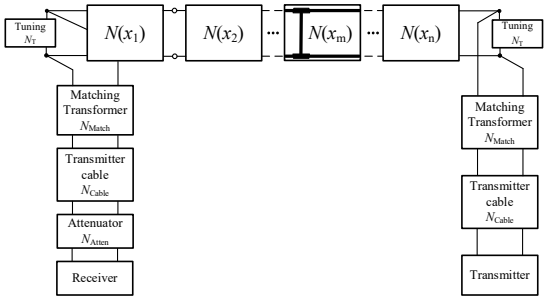

Fig. 1. ZPW-2000A four-port network model.

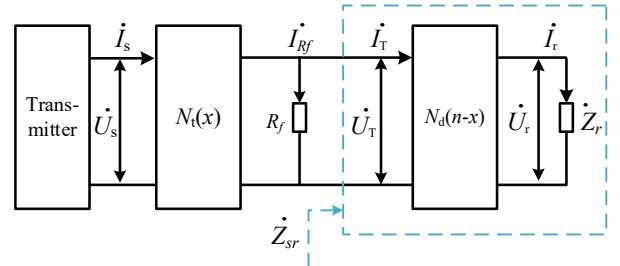

Fig. 2. Cab signal voltage model.

The equivalent model of cab signal voltage in shunting state is shown in Figure $2 . \dot{Z}_{s r}$ is the apparent impedance of the receiving end, which can be expressed as:

$$
\dot{Z}_{s r}=\frac{N_{d 11}(n-x) \dot{Z}_{r}+N_{d 12}(n-x)}{N_{d 21}(n-x) \dot{Z}_{r}+N_{d 22}(n-x)}
$$

The cab short circuit current $\dot{I}_{R f}(x)$ is:

$$
\dot{I}_{R f}(x)=\frac{\dot{U}_{s}}{N_{t 11}(x) \cdot R_{f}+N_{t 12}(x)\left(R_{f}+\dot{Z}_{s r}(x)\right) / \dot{Z}_{s r}(x)}
$$

The following quantitative relationship between the cab signal receiving voltage and current is[9]:

$$
\dot{V}_{R f}(x)=\frac{200}{255} \dot{I}_{R f}(x)
$$

Without loss of generality, take the track circuit length $L_{x}$ as $1200 \mathrm{~m}$, the compensation capacitor $C$ as $25 \mu \mathrm{F}$, the cable transmission length $L_{d}$ as $10 \mathrm{~km}$. The transmission voltage is three levels $(140 \mathrm{~V})$ and the ballast resistance $R_{d}$ as $2 \Omega \cdot \mathrm{km}$. Through MATLAB software simulation, the cab signal receiving voltage under different carrier frequencies is obtained, as shown in Figure 3. It can be seen that due to the influence of the compensation capacitor, the received voltage value of the cab signal fluctuates and rises from the receiving end, and a turning point appears at each compensation capacitor. Its function is to reduce line loss, increase transmission distance, and improve the reliability of information exchange between vehicles and ground. In addition, due to the difference in resistance 
value, inductance value and compensation capacitor spacing of the track circuit in different carrier frequency sections, the current received by the cab is lower in the section with higher carrier frequency.

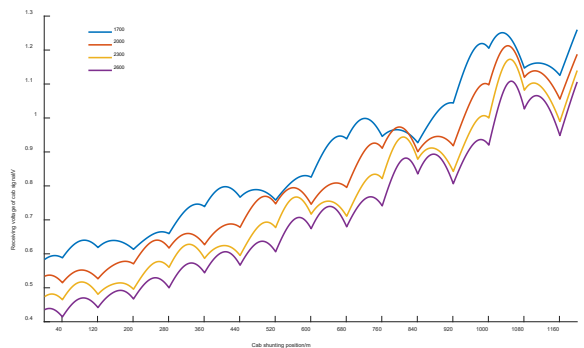

Fig. 3. Simulation results of cab signal receiving voltage.

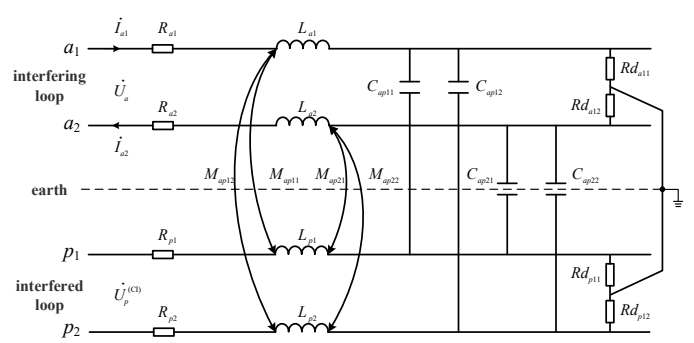

Fig. 4. the calculation model of adjacent line coupling interference.

\subsection{Theoretical calculation of adjacent line interference modelling}

Figure 4 shows the calculation model of adjacent line coupling interference. The calculation formula of mutual inductance coefficient $M_{a p}$ of parallel running railway lines $a$ and $p$ is as follows:

$$
M_{a p}=\left(k \times 200 \times \ln \frac{D_{a p 12} \times D_{a p 21}}{D_{a p 11} \times D_{a p 22}}\right) \times 10^{-6}
$$

In the formula, $k$ is the correction factor, which is related to the carrier frequency. Since the carrier frequency of the signal flowing through the rail is much less than $4 \mathrm{MHz}$, the mutual impedance between two parallel track circuits $a$ and $p$ can be calculated according to Carson's theory [10] as:

$$
Z_{a p}=j w M_{a p}=\pi^{2} f \times 10^{-4}+j 0.00289 f \ln \frac{D_{g}}{D_{a p}}
$$

Among them, $f$ is the carrier frequency of the signal flowing through the rail; $D_{g}=660 \sqrt{\sigma_{d} / f}$ is the equivalent depth of the line, $\sigma_{d}$ is the earth conductivity (take the average, $\left.\sigma_{d}=10^{-2} \Omega \cdot \mathrm{m}\right)$. Then the mutual inductance voltage generated can be obtained as follows:

$$
\dot{U}_{p}^{(\mathrm{MI})}=Z_{a p} \dot{I}_{a} D_{a \mathrm{p}}
$$

Capacitance coupling is also called electric field coupling. For the convenience of modelling, capacitance coupling and ballast resistance conduction are combined for analysis in Fig. 5. The interference voltage $\dot{U}_{p}^{(\mathrm{CR})}$ generated by capacitance coupling and ballast resistance leakage conduction interference in the interfered loop is as follows:

$$
\dot{U}_{p}^{(\mathrm{CR})}=\dot{I}_{z 1} \dot{Z}_{c r 2}-\dot{I}_{z 2} \dot{Z}_{c r 4}
$$

Adjacent line interference will directly affect the value of cab signal entry current in the series circuit. $\dot{U}_{G}$ is the interference voltage of the interfering loop to the interfered loop. 


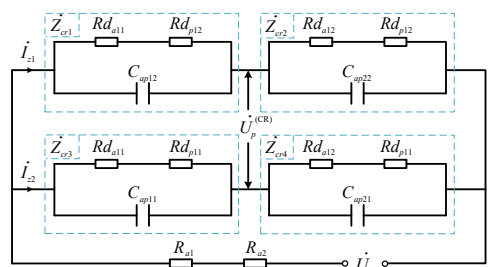

Fig. 5. Equivalent circuit of capacitance coupling and ballast resistance conduction.

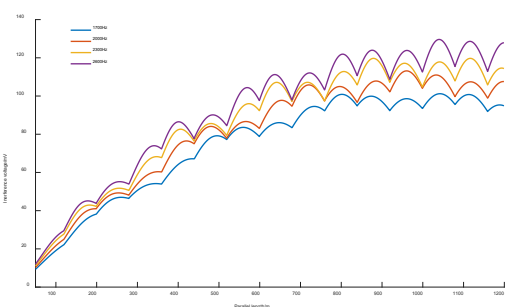

Fig. 6. Interference voltage with parallel length under different carrier frequencies.

In order to study the influence of carrier frequency on adjacent line coupling interference voltage, the interference voltage of the interfered loop is simulated under the conditions of $1700 \mathrm{~Hz}, 2000 \mathrm{~Hz}, 2300 \mathrm{hz}$ and $2600 \mathrm{~Hz}$ carrier frequency respectively of the interfering loop. The simulation results are shown in Figure 6. It can be seen that when the carrier frequency is $2600 \mathrm{~Hz}$, the interference voltage to the interfered loop is the largest.

\section{Index evaluation and safety protection of adjacent line interference}

\subsection{Index evaluation of adjacent line interference}

The interference of adjacent lines is easy to cause the wrong action of on-board equipment, so it is necessary to evaluate the interference of adjacent lines according to the safety index of cab. When the interfered loop is in the adjustment state, the interference of adjacent lines shall not exceed the sensitivity of cab signal to prevent the "red light band" phenomenon; when the interfered loop is in shunting state, the interference from adjacent lines shall not exceed the reliable falling value of cab signal to prevent signal upgrading accident. Under the above calculation conditions, the maximum parallel length is shown in Table 1. If it exceeds the recommended value, it may cause equipment misoperation and increase the hidden danger of driving safety.

Table 1. Maximum parallel length under different carrier frequencies.

\begin{tabular}{ccccc}
\hline $\begin{array}{c}\text { carrier } \\
\text { frequency } \\
(\mathrm{Hz})\end{array}$ & $\begin{array}{c}\text { adjustment state } \\
\text { Maximum }\end{array}$ & $\begin{array}{c}\text { Maximum } \\
\text { parallel } \\
\text { length(m) }\end{array}$ & $\begin{array}{c}\text { shunting state } \\
\text { Maximum } \\
\text { interference(m) }\end{array}$ & $\begin{array}{c}\text { Maximum parallel } \\
\text { length(m) }\end{array}$ \\
\hline 1700 & 92.5 & 712 & 69.4 & 446 \\
2000 & 92.5 & 623 & 69.4 & 383 \\
2300 & 92.5 & 551 & 69.4 & 363 \\
2600 & 92.5 & 533 & 69.4 & 314 \\
\hline
\end{tabular}

\subsection{Suggestions on adjacent line interference protection}

The existence of adjacent line interference greatly improves the probability of locomotive signal upgrading or code dropping, and increases the hidden danger of train operation. Based on the above theoretical analysis, the following measures can be taken to protect the engineering design.

(1) Try to avoid the configuration of the same carrier frequency between adjacent lines; 
(2) Appropriately increase the line spacing of lines with the same carrier frequency;

(3) The tuning area is staggered to reduce the parallel length;

(4) The spacing of compensating capacitors is different to reduce the area of equivalent loop.

\section{Conclusion}

This paper focuses on the problem of electromagnetic interference between adjacent lines in multi-line parallel section of high-speed railway. The specific conclusions are as follows:

(1) In the context of high-speed railway, the main factors causing adjacent line interference is rail mutual inductance coupling, which can reach $100 \mathrm{mV}$ under the most unfavorable conditions.

(2) From the perspective of normal operation of cab equipment, it is suggested to increase line spacing and reduce the parallel length between lines to reduce the interference of adjacent lines.

\section{References}

1. Liu C, Yang S, Cui Y, et al. Optimization method of switch jumper setting based on strategies for reducing conductive interference in railway[J]. Proceedings of the Institution of Mechanical Engineers Part F Journal of Rail and Rapid Transit, 2020:095440972095130.

2. Liu C, Yang S, Cui Y, et al. Quantitative analysis on coupling of traction current into cab signaling in electrified railways[J]. Railway Engineering Science, 2020, 28(3):275289.

3. Lucca, Giovanni. Influence of railway line characteristics in inductive interference on railway track circuits[J]. IET Sci-ence, Measurement Technology, 2019.

4. E. M. Tarasov et al. A method for determination of the matrix coefficients of the A parameters of a multipole that simulates the impact of the adjacent track circuit[J]. Russian Electrical Engineering, 2017, 88(3) : 99-104.

5. Manakov A D, Kudryavtsev V A, Os'Minin A T. Methods of determining the interference current in rails of double sub-way track circuits[J]. Russian Electrical Engineering, 2016, 87(5):282-285.

6. ZHAO Cuiqin, WU Xiaochun. Analysis of the Interference Effect of Rail Mutual Impedance on the Information Trans-mission of Adjacent Lines[J]. Journal of Lanzhou Jiaotong University, 2020, 039(001):73-79. (in Chinese)

7. LI Yuequan. Analysis and Solution of Same Frequency Interference Between Multiline Parallel Sections[J]. Value Engineering, 2018,37(028):189-191. (in Chinese)

8. LIU Jialiang, BI Hongjun, YANG Shiwu etc. Simulation Research on Interference of Adjacent Section of ZPW-2000 Non-insulated Track Circuit[J]. Railway computer application, 2014,23(05):45-48. (in Chinese)

9. Ministry of Railways of the People's Republic of China. Technical Standards for Railway Signal Maintenance Rules I[M]. 3rd edition. Beijing: China Railway Press, 2014: 54-59. (in Chinese)

10. DU Xuelong, WANG Zhixin, ZOU Jun. Simplified formula for fast calculation of mutual impedance of high-speed rail-way track circuit considering the influence of the earth[J]. Journal of Electrotechnical Technology, 2016,31(04):1-6. (in Chinese) 